\title{
EARLY POSTNATAL DEVELOPMENT OF LAMINAR CHARACTERISTICS IN THE DORSAL LATERAL GENICULATE NUCLEUS OF THE TREE SHREW ${ }^{1}$
}

\author{
J. K. BRUNSO-BECHTOLD ${ }^{2}$ AND V. A. CASAGRANDE
}

Departments of Anatomy and Psychology, Vanderbilt University, Nashville, Tennessee 37232

Received September 28, 1981; Revised December 21, 1981; Accepted December 30, 1981

\begin{abstract}
Three characteristics distinguish the six layers of the adult tree shrew dorsal lateral geniculate nucleus (LGN). First, interlaminar spaces divide the nucleus into cell layers. Second, input from the two eyes projects to the nucleus such that two layers (1 and 5) receive input from the ipsilateral retina and four layers $(2,3,4$, and 6$)$ receive input from the contralateral retina. Finally, distinct cytological features characterize individual layers. In this report, we describe the postnatal development of LGN layers in the tree shrew in terms of the development of these three characteristics.

At birth, the nucleus appears homogeneous in Nissl-stained sections. Thus, no interlaminar spaces are present and all cells look similar in shape, size, and staining intensity. However, autoradiographic data show that, at birth, retinal afferents are segregated in an adult-like pattern. Interlaminar spaces begin to be evident between layers innervated by opposite eyes on postnatal day 2 . Several days later, interlaminar spaces between layers innervated by the same eye (i.e., the borders of layer 3) appear, while the others continue to widen. Although some cytological maturation begins before interlaminar space formation, it is not until interlaminar spaces are apparent that features such as differential staining intensity and cell size can be used to distinguish individual layers.

The results suggest that the three characteristics that define LGN layers in the tree shrew may be temporally separate events in the developing nucleus. Thus, retinal afferents are segregated prior to interlaminar space formation which, in turn, is initiated prior to final maturation of the cytological features that characterize the cell layers. This may indicate a degree of developmental independence among these maturational events.
\end{abstract}

In most species with a laminated dorsal lateral geniculate nucleus (LGN), the layers develop prenatally (Gilbert, 1934; Cooper, 1945; Moskowitz and Noback, 1962; Rakic, 1977a, b; Kalil, 1978; Hitchcock and Hickey, 1980). This fact has made it extremely difficult to follow the normal sequence of developmental events leading to layer formation as well as to manipulate those events experimentally.

In the tree shrew, all LGN cell layers form postnatally (Zilles, 1978; Brunso-Bechtold and Casagrande, 1980, 1981; Rager el al., 1980), providing an opportunity to observe and manipulate their developmental history. The

\footnotetext{
'We wish to thank Elizabeth Birecree for technical assistance in all aspects of this project, Brenda Lair and Vera Henley for typing the manuscript, and Dr. Mike Sesma for careful reading of the manuscript. This research was sponsored by Public Health Service Research Grants EY01778 and EY03881 and Public Health Service Research Career Development Award 1 K04-EY00223-01 to V. A. C. and Public Health Service Postdoctoral Fellowship 1F32NS06206 01 to J. K. B. -B.

${ }^{2}$ To whom correspondence should be addressed.
}

tree shrew LGN is also especially suitable for the study of laminar development for several other reasons. First, in the adult LGN, borders between the six layers are quite clear (see Fig. 1), allowing for easy identification. Second, the LGN of the tree shrew contains two types of interlaminar spaces, those between layers receiving input from the same eye and those between layers receiving input from opposite eyes. This fact provides an opportunity to compare the development of interlaminar spaces between layers that have segregated for presumably different functional reasons. Finally, many details of the connections, physiology, and relative cell sizes of IGN layers in the adult tree shrew are known, allowing for easy comparison of the mature and immature nucleus (Campbell et al., 1967; Carey et al., 1979; Casagrande, 1974; Casagrande et al., 1978; Casagrande and Harting, 1975; Hubel, 1975; Brauer et al., 1981; Glickstein, 1967; Harting et al., 1973; Laemle, 1968; Sherman et al., 1974; Norton et al., 1977; Tigges, 1966; Snyder and Diamond, 1968; Fitzpatrick et al., 1980; Birecree et al., 1980).

In the present communication, it is our goal to define 
the normal sequence of events leading to laminar formation in the tree shrew LGN. We will focus on three developmental events: the segregation of retinal afferents into layers, the formation of interlaminar spaces between adjacent cell layers, and the cytological maturation of the cells within those layers. Our results provide evidence that, while retinal afferents from the two eyes are segregated at or before birth, interlaminar space formation and final cell maturation occur postnatally.

\section{Materials and Methods}

Twenty-one tree shrews (Tupaia glis) were used in the present study. Of these animals, 19 were perfused during the first 2 postnatal weeks and 2 were perfused at maturity. Fifteen of the infants and 1 adult were used to study the normal cytoarchitecture of the LGN, and the remaining 4 infants and 1 adult were used to investigate the pattern of retinal projections.

All infants were born in our colony after an average gestational period of 43 days. Each litter consisted of 1 to 3 pups whose body size at birth varied both between and within individual litters. Although no effort was made to control for initial body size, our practice of hand rearing eliminated increases in size differences which can result from competition for, or variation in, maternally provided nutrition. Individual differences in the stage of development at particular postnatal ages also were seen in geniculate development especially at the more advanced stages. Consequently, although we specify the time of birth and refer to the animals by age, our interest is not in the absolute time of occurrence of events in geniculate development but rather in the occurrence of those events relative to one another.

Throughout the present report, we will refer to the first $24 \mathrm{hr}$ after birth as postnatal day (PND) 0 , and thus, an animal designated as PND 3 will have completed the 3rd day of life. In order to study the normal cytoarchitecture of the tree shrew LGN, the brains of animals perfused on PND 0 through PND 8 as well as PND 14 were processed using celloidin embedding techniques. In addition, animals in this series were sacrificed on PNDs $1,3,4$, and 5 and processed using paraffin techniques. Each animal was first anesthetized and then perfused transcardially with $50 \mathrm{ml}$ of $10 \%$ buffered formalin, and the brain was stored in $10 \%$ formalin for at least 1 week. For celloidin processing, the brain was cut along the midsagittal line and dehydrated in a series of alcohols, and each half was embedded separately. One half was sectioned in the coronal plane, and the other in the horizontal plane, at either 20 or $30 \mu \mathrm{m}$, and every other section was stained with thionin. For paraffin processing, the entire brain was dehydrated, embedded in paraffin, and cut in the horizontal plane at $20 \mu \mathrm{m}$, and every fifth section was stained with cresyl violet. The LGN in the sections of both series was studied and its cytoarchitecture was reconstructed with the aid of a Zeiss camera lucida drawing tube. Measures of volume and area were calculated from outline drawings using a Bioquant II computerized image analysis system (E. Leitz).

In order to determine retinal projections to the LGN, 4 infants were given intraocular injections of $5 \mu \mathrm{l}$ of $\left[{ }^{3} \mathrm{H}\right]$ proline (New England Nuclear; specific activity, $20 \mathrm{Ci}$ / mmol) on PND 0 . Two of these animals were perfused 6 $\mathrm{hr}$ after the injection, and 2 were sacrificed $12 \mathrm{hr}$ after the injection. For comparison, 1 adult tree shrew received an intraocular injection of $5 \mu \mathrm{l}$ of $\left[{ }^{3} \mathrm{H}\right]$ proline $(500 \mu \mathrm{Ci}$ in saline) and was allowed to survive for $72 \mathrm{hr}$. Following perfusion and brain removal as described above, the brains of 2 of the infants were embedded in gelatin. These two infant brains and the adult brain then were cut horizontally as $30-\mu \mathrm{m}$ frozen sections. The other two infant brains were embedded in paraffin and cut horizontally at $20 \mu \mathrm{m}$. All sections were processed for routine autoradiography and counterstained with cresyl violet as described previously (Cowan et al., 1972; Edwards, 1972; Casagrande et al., 1978).

\section{Results}

Before presenting our results, it is important to clarify the term laminar differentiation. This term can be used to define several different aspects of the formation of layers. For example, laminar differentiation can refer to the acquisition of layers defined by afferent input, i.e., the segregation of afferents into distinct layers. It also can refer to the acquisition of layers defined by cytoarchitectural features, i.e., characteristic inter-relationships of cell groups, such as interlaminar spaces and packing density. Finally, laminar differentiation can refer to the acquisition of layers defined by cytological features, i.e., the characteristics of the cells themselves, such as cell size, shape, or orientation and amount of Nissl substance.

Obviously, the distinction between the definition of layers by cytoarchitectural and cytological features is somewhat arbitrary. Nevertheless, our present and past work (Brunso-Bechtold and Casagrande, 1980, 1981) suggests that these features of laminar formation, as well as the definition of layers by segregation of afferent input, are temporally separate and, in some cases, independent events. Thus, in the presentation that follows, we compare each stage of LGN development in terms of these three aspects of laminar differentiation.

The mature tree shrew LGN. For comparison with the immature cases, we will first describe the anatomy of the mature tree shrew LGN. Figure 1 shows a horizontal Nissl-stained section through the LGN of a mature tree shrew, while Figure 2, $A$ and $B$, shows the adult pattern of retinal innervation of the nucleus. The horizontal plane most clearly reveals the laminar organization of the nucleus, and consequently, this is the plane that we use throughout the paper for illustration.

The nucleus in the mature animal is shaped somewhat like a curved, elongated sphere approximately $1 \times 1 \mathrm{~mm}$ in the rostrocaudal and mediolateral planes and $4 \mathrm{~mm}$ in the dorsoventral plane. It is bounded laterally by the optic tract and medially by the capsular fibers of the optic radiations. The latter fibers divide it from the pulvinar at its posterior boundary and from the ventral lateral geniculate nucleus at its anterior boundary. The nucleus is composed of six layers which are numbered from medial to lateral with layer 6 lying closest to the optic tract (see Fig. 1). Although the quantitative and qualitative differences between these layers have been described by us and other investigators (see Casagrande et al., 1978, for review), it is worthwhile here to recapitulate some of the major distinguishing features of the layers for ease of comparison with the developing LGN. 


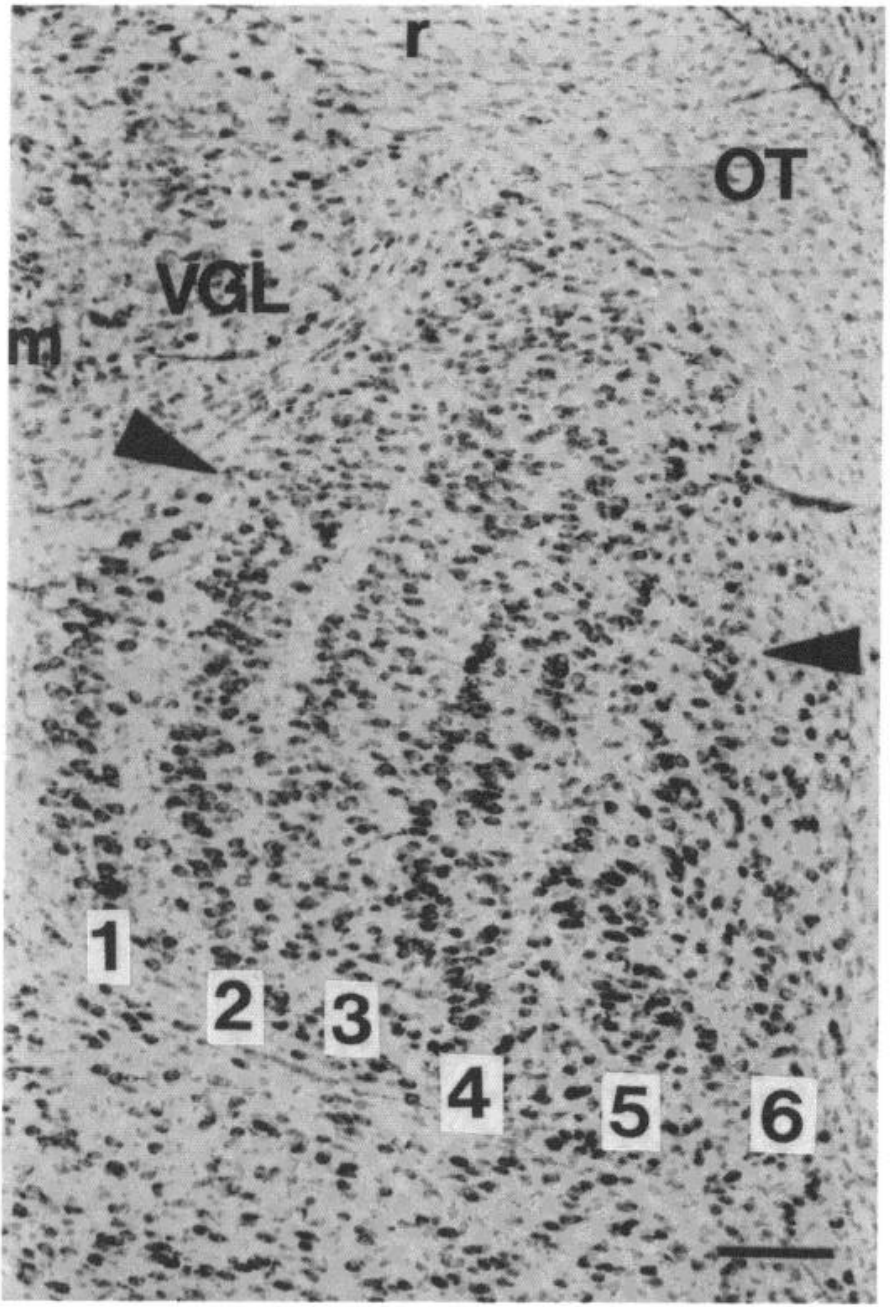

Figure $1 .^{3}$ Horizontal section through the LGN of a mature tree shrew. Of the six layers, layers 1 and 5 are innervated by the ipsilateral eye and layers $2,3,4$, and 6 are innervated by the contralateral eye. The layers are separated by cell-free interlaminar spaces and, in addition, are distinguished by different cytoarchitectural and cytological features (see text). Only the contralaterally innervated layers are present in the monocular segment which is above the arrowheads. Bar, $0.1 \mathrm{~mm}$.

Based both upon retinal afferents and cytology, the six layers of the nucleus can best be grouped into two matched pairs $(1,2$ and 4,5$)$ and two unique layers $(3$ and 6). In terms of retinal afferents, each matched pair contains one layer innervated by each eye such that layers 1 and 5 receive ipsilateral retinal input, and 2 and 4 receive contralateral input. The remaining two layers, 3 and 6, receive contralateral retinal input (see Fig. 2, $A$ and $B$ ). Cytologically, layers 1 and 2 appear as a matched pair of well defined, narrow layers with medium size spindle-shaped cells which are oriented perpendicular to laminar borders. Layers 4 and 5 also contain spindleshaped cells oriented in a similar fashion; however, these layers are wider and contain more rounded, darkly staining, and slightly larger somata. In addition, cells in layer 4 frequently appear to be organized into sublaminae.

\footnotetext{
${ }^{3}$ The abbreviations used on the figures are: $\mathrm{m}$, medial; OT, optic tract; r, rostral; VGL, ventral lateral geniculate nucleus.
}

Layer 3 contains the smallest and most lightly staining cells in the nucleus and these are either polygonal or spindle shaped and are oriented as described for the other layers. Layer 6 forms a thin shell around the lateral margin of the nucleus. It contains a mixture of cells of different sizes and shapes; some of the cells are oriented parallel rather than perpendicular to laminar borders, and the cells in general appear more scattered than elsewhere in the nucleus.

The six-layer pattern that we have described is most distinct in the middle one-third of the dorsoventral and rostrocaudal extent of the nucleus. Nevertheless, some laminar borders can be seen in other parts of the nucleus except where the four contralaterally innervated layers $(2,3,4$, and 6) merge in the monocular segment (see arrowheads in Fig. 1) located rostrally and ventrally within the nucleus.

PNDs 0 to 1: Period prior to interlaminar space formation. At birth, the tree shrew LGN lies in about the same position in the dorsal thalamus as in the adult. Although the LGN of the neonatal tree shrew is much smaller and its shape is somewhat different from that of the adult (see below), the nucleus does not rotate during development. Such developmental rotations have been described in the carnivore and primate (Rakic, 1977a; Kalil, 1978; Hitchcock and Hickey, 1980; Shatz and DiBerardino, 1980; Linden et al., 1981).

From birth through approximately PND 1, no cell layers or interlaminar spaces are present in any part of the nucleus (see Figs. $3 C$ and $4 A$ ), and while some sections reveal occasional hints of differential packing density, no laminar pattern is apparent.

In contrast to the lack of cytoarchitectural subdivision into layers (Fig. $3 C$ ), the retinal afferents to the LGN are clearly laminated at birth (Fig. $3, A$ and $B$ ). This segregation of afferents is evident in the dark-field photomicrographs of label in the LGN of a tree shrew which received a monocular injection of $\left[{ }^{3} \mathrm{H}\right]$ proline on PND 0 and was perfused $12 \mathrm{hr}$ later, early on PND 1 (i.e., prior to cytoarchitectural differentiation). This same pattern of label also is seen in animals which received eye injections immediately after birth and were perfused $6 \mathrm{hr}$ later, still on PND 0 . With our present material, it is difficult to be certain if the laminar segregation of retinal afferents is complete at birth. In fact, in some sections from the LGNs of animals sacrificed either on PND 0 or 1 , one has the impression that afferent layers 2,3 , and 4 are not as clearly segregated as in the adult and that background is higher in some of the presumptive interlaminar regions, e.g., between layers 2 and 3 and layers 3 and 4 (compare Figs. $2 A$ and $3 A$ ). Whether this is an artifact due to the absence of interlaminar spaces or truly represents a less mature state would be difficult to say without experiments involving separate markers for both left and right eye afferents. The point, however, remains that all afferent layers are recognizable as separate entities at birth, while none of the cell layers are apparent.

In addition to appearing cytoarchitecturally immature, the PND 0 to PND $1 \mathrm{LGN}$ is cytologically immature as well. Layers cannot be distinguished based upon cytological criteria, and in general, cells have an immature appearance with indistinct nuclear borders, multiple nucleoli, and little cytoplasm. Figure $5 A$ shows a high power 

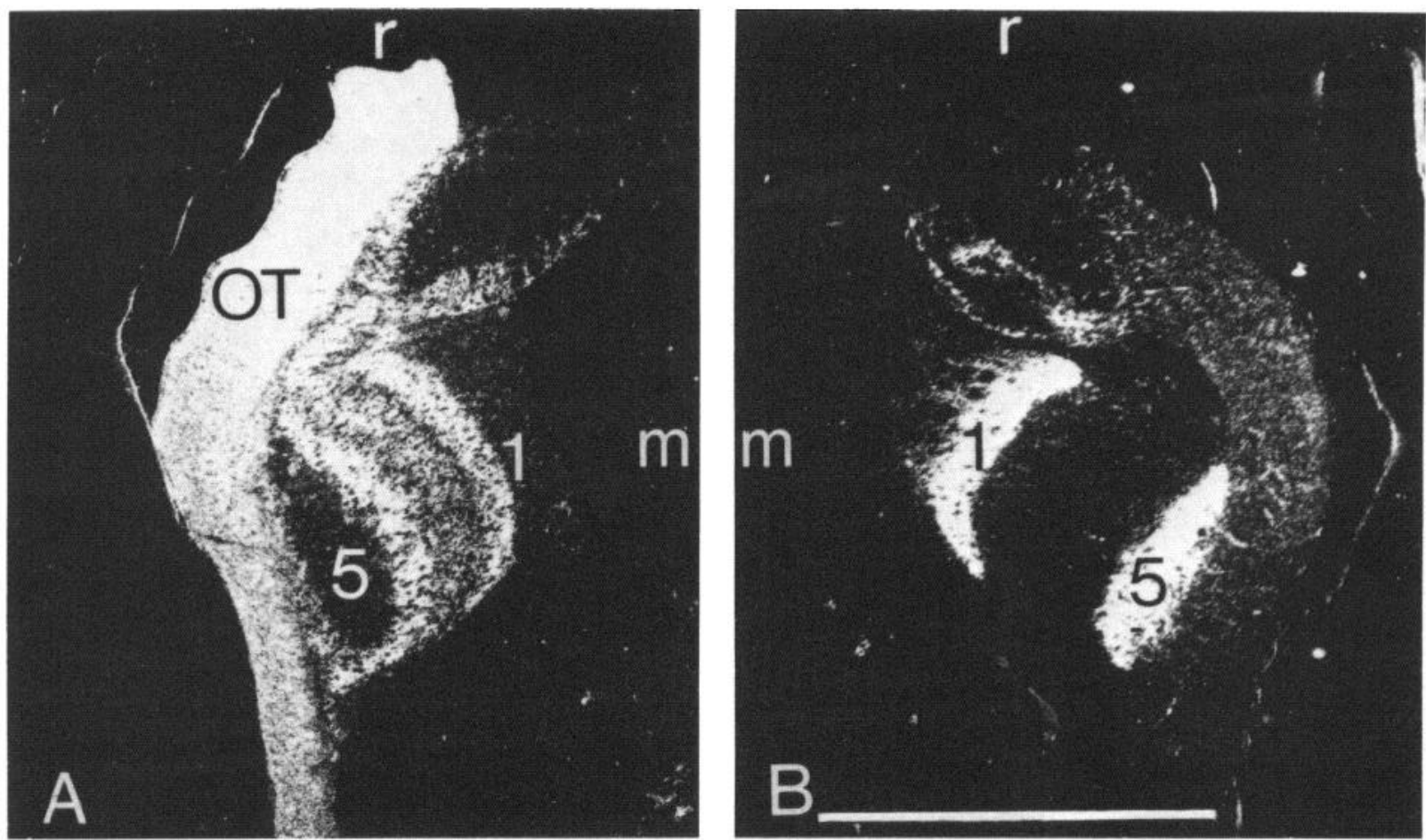

Figure 2. Low power dark-field photomicrograph of the LGN in a mature tree shrew which received a monocular injection of $\left[{ }^{3} \mathrm{H}\right]$ proline $48 \mathrm{hr}$ before sacrifice. $A$, Contralateral to the injected eye; $B$, ipsilateral to the injected eye. Note that all layers except 1 and 5 are filled with label and tracer in the contralateral LGN, whereas only those two layers are filled with labeled tracer in the ipsilateral nucleus. Bar, $1 \mathrm{~mm}$.
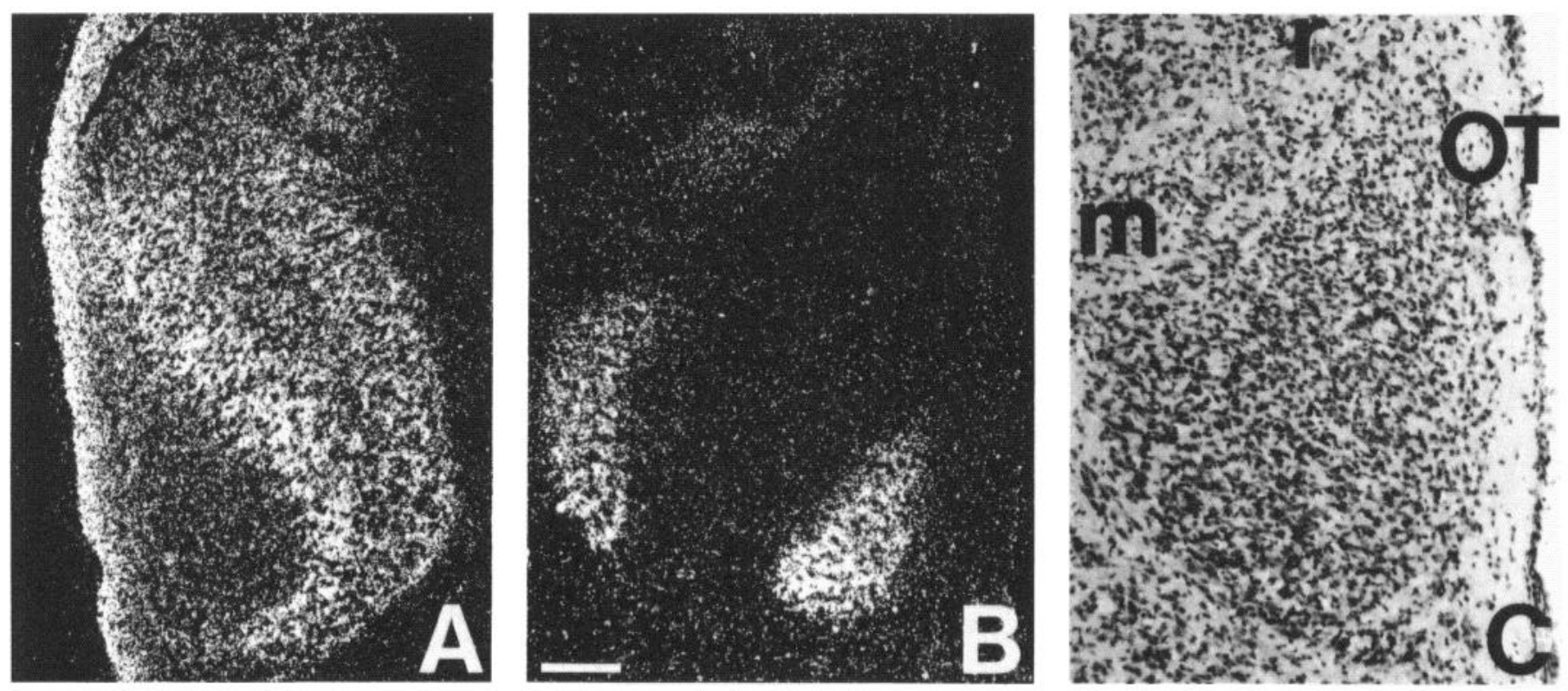

Figure 3. Horizontal sections through the LGN of an infant tree shrew which received a monocular injection of $\left[{ }^{3} \mathrm{H}\right] \mathrm{proline}$ on PND 0 and was sacrificed $12 \mathrm{hr}$ later, early on PND 1. A, Dark-field photomicrograph of the LGN contralateral to the injected eye; $B$, dark-field photomicrograph of the LGN ipsilateral to the injected eye; $C$, light-field photomicrograph of the same section shown in $B$. Note that layers of retinogeniculate termination in the neonate are very similar to those in the adult (see Fig. 2), whereas no cytoarchitectural or cytological layers can be seen in the neonate. Bar, $0.1 \mathrm{~mm}$.

photomicrograph of cells at the medial border of a PND 0 tree shrew LGN. Typically, however, cells in the middle one-third of the nucleus are at somewhat more advanced stages of development; these cells may be oriented, and in addition, contain more cytoplasm. In contrast, Figure
$5 B$ shows cells at the medial border (layer 1 ) of a mature tree shrew LGN. In these cells, the cytoplasm is more apparent and clearly staining, and the centrally located nucleus is well defined and only one or two clear nucleoli are present. 

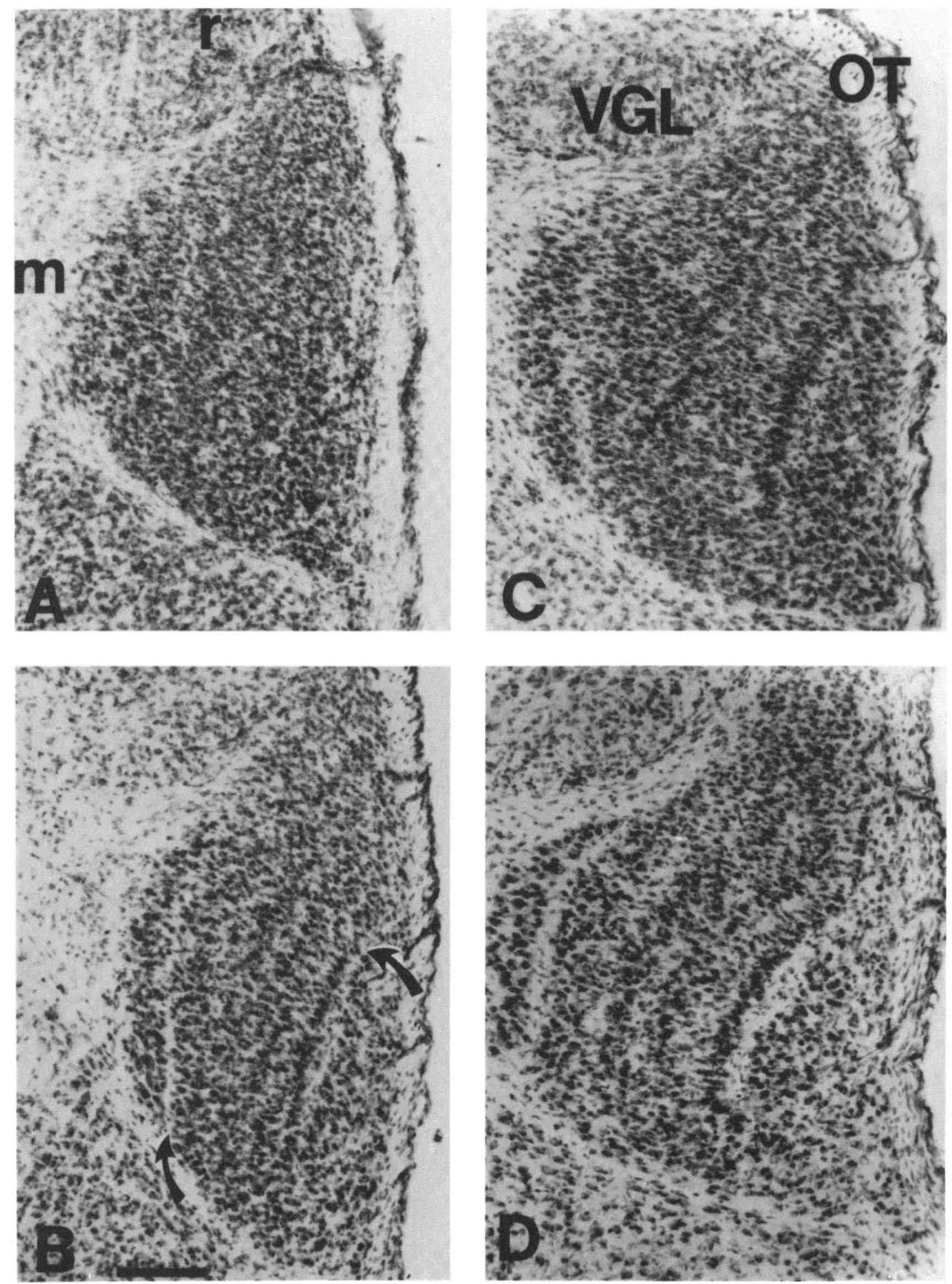

Figure 4. Horizontal sections through the LGN of tree shrews at various stages of laminar development. $A$, PND 0 , prior to any cytoarchitectural or cytological lamination. $B$, PND 3, during the period of formation of interlaminar spaces between layers innervated by opposite eyes. The arrows indicate the beginnings of interlaminar spaces between layers 1 and 2 and layers 4 and 5. In addition to interlaminar spaces, other cytoarchitectural features, such as the increase in cell density at the outer edge of presumptive layer 4, begin to appear at this time. $C$, PND 6, during the period of formation of interlaminar spaces between layers innervated by the same eye. $D$, PND 8, later in the same developmental period as $C$. Bar, $0.1 \mathrm{~mm}$. 

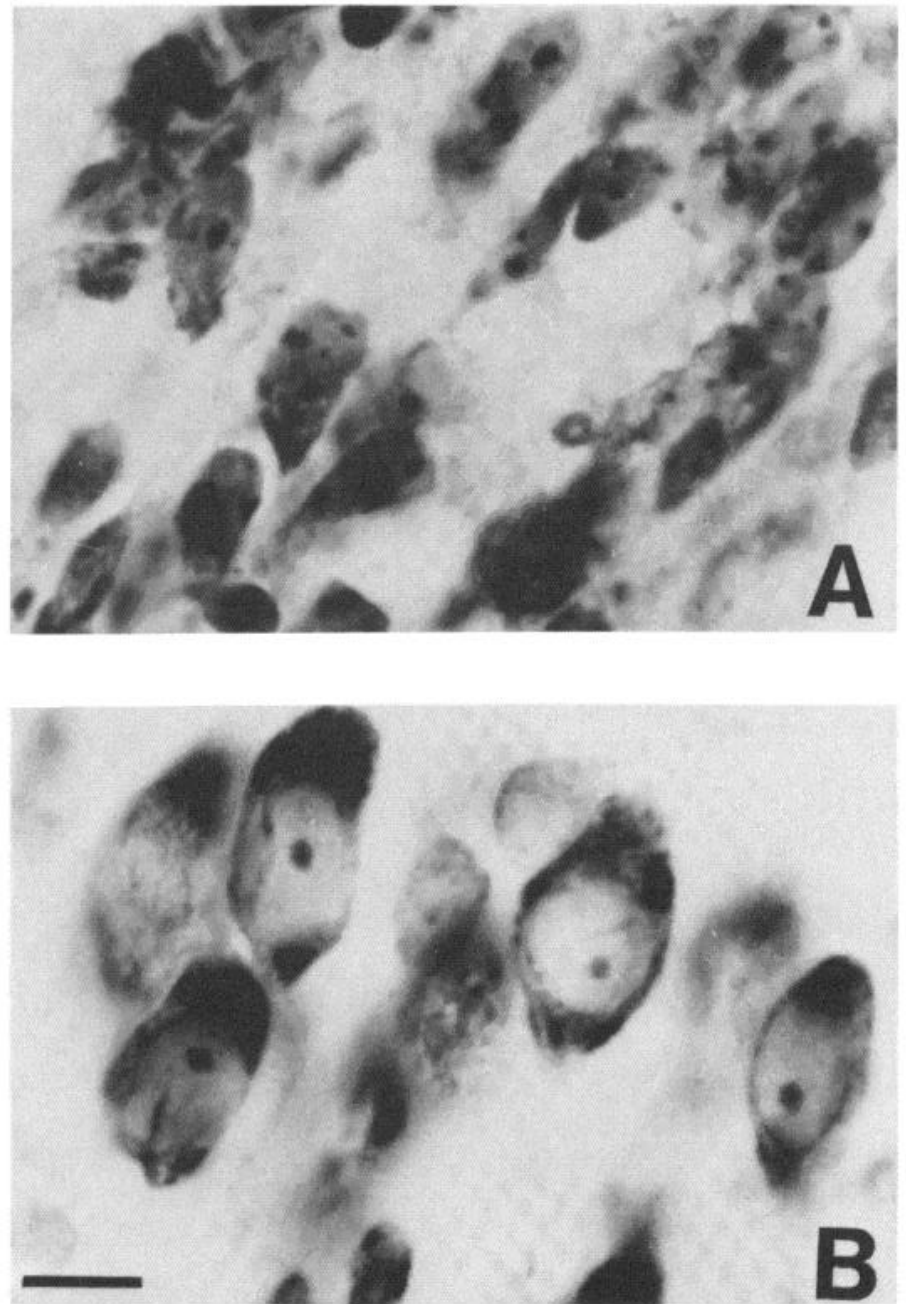

Figure 5. High power photomicrographs of LGN cells at different stages of development. $A$, Poorly differentiated cells from the medial margin of a tree shrew LGN on postnatal day 0 . Note the pale staining, scant cytoplasm, the indistinct nuclear borders, and multiple nucleoli. $B$, Well differentiated cells from the medial margin (layer 1) of a mature tree shrew LGN. Note the dark staining, abundant cytoplasm, the clear nuclear borders, and the single or paired nucleoli. Bar, $0.01 \mathrm{~mm}$.

PNDs 2 to 4: Period of formation of interlaminar spaces between layers innervated by opposite eyes. During this period, interlaminar spaces between LGN cell layers begin to form. They can first be recognized on PND 2 roughly in the middle of the nucleus where the adult LGN also exhibits the most distinct laminar boundaries. Retinotopically, this region would correspond to the region representing visual space within a $10^{\circ}$ to $20^{\circ}$ radius of the area centralis (T. T. Norton and V. A. Casagrande, unpublished observation).

Interlaminar spaces appear first as a few scattered cellfree zones between layers innervated by opposite eyes, particularly layers 1 and 2 and layers 4 and 5 . Figure $4 B$ illustrates the developing interlaminar spaces between layers 1 and 2 and 4 and 5 on PND 3. Suggestions of an interlaminar space between layers 5 and 6 also are becoming apparent at this time. This latter interlaminar space, however, is indistinct even in the adult and, consequently, is difficult to follow developmentally. Also present during this period are hints of decreases in cell density within prospective interlaminar regions dividing the contralaterally innervated layers (see Fig. $4 B$ ). Although unequivocal laminar identification is obviously impossible during early development, a careful comparison of the mature LGN (Fig. 1) with this figure and ensuing ones should support our distinctions. The spaces, however, do not become distinct for nearly another week.

During this same period, cytological maturation progresses such that some somata exhibit the spindle shape and orientation characteristic of mature cells. Even these more developmentally advanced cells, however, still appear relatively immature when compared to those in the adult and exhibit multiple nucleoli and relatively little cytoplasm. Moreover, cell size and relative staining intensity still appear uniform throughout the nucleus.

PNDs 5 to 8: Period of formation of interlaminar spaces between layers innervated by the same eye. The slight variations in packing density which can be seen between layers innervated by the same eye (i.e., between contralaterally innervated layers 2 and 3 and layers 3 and 4) on PND 2 gradually increase and scattered cell-free spaces can be seen by PND 6 (Fig. 4C). These spaces coalesce, and by PND 8, there are rudimentary interlaminar spaces between layers 2 and 3 and layers 3 and 4 (Fig. $4 D$ ). These spaces begin in approximately the middle third of the nucleus. Moreover, by this stage of development, the interlaminar spaces between layers innervated by opposite eyes have extended further through the nucleus.

In addition to interlaminar spaces, other cytoarchitectural features are beginning to be apparent. The dense packing of cells which is characteristic of layer 4 begins by PND 3 and is clearly present by PND 6 (Fig. $4 C$ ). Moreover, the characteristic division of this layer into two sublaminae can be seen by PND 8 (Fig. $4 D$ ). Finally, by PND 8 , the general width and shape of the layers is beginning to resemble that of the adult (compare Fig. 1 with Fig. 4D).

Several characteristic cytological features also mature rapidly during this period. By PND 8, the LGN cells are well differentiated and clearly oriented perpendicular to the interlaminar spaces, although the orientation of cells in layer 5 seems to lag somewhat behind that of cells in the rest of the layers. In addition, the cells in layer 6 as well as in layer 3 appear smaller than those in other layers as is the case in the adult. While it is not obvious in Figure $4 C$, the cells in layers 3 and 4 are taking on their pale and dark characteristics, respectively.

PND 8 and beyond: Final stages of LGN maturation. By PND 8, the LGN of some tree shrews exhibits an essentially adult-like structure. In others, the nucleus does not reach this stage until as late as PND 14 or possibly beyond. Comparison of the nucleus at 2 weeks with that of an adult suggests that the nucleus continues to enlarge, with cells increasing in size and laminar borders becoming sharper between 2 weeks and 3 months (sexual maturity).

Our preliminary measures of the overall volume of the adult nucleus suggest that it is more than 5 times larger by volume than that of the PND 0 infant. Although we have not as yet measured cell packing density, Zilles' (1978) results indicate that, in the adult or 3-month-old 
tree shrew, the LGN cells are 5 times less densely packed than on PND 0 . As mentioned earlier, however, the overall growth of the nucleus does not cause it to change its general orientation. Rather our measures show that the ratio of the area of the nucleus at its widest extent to its dorsoventral length changes from approximately 1:7 in the PND 0 infant to $1: 5$ in the adult.

\section{Discussion}

Temporal relationship of developmental events within the LGN. As we have stated, interlaminar spaces are absent in the tree shrew LGN at birth. This observation is consistent with the findings of Rager et al. (1980) who also have studied the developing tree shrew LGN. Our results further demonstrate that these interlaminar spaces develop during the ensuing 2 weeks. It is noteworthy that the formation of interlaminar spaces is preceded at least several days by the segregation of retinal afferents; at birth, the fibers from the ipsilateral eye project to the general region of future cell layers 1 and 5 and those from the contralateral eye project to the region of future cell layers $2,3,4$, and 6 . Following monocular injections of horseradish peroxidase on the 1st postnatal day in the tree shrew, Rager et al. (1980) report anterograde transport to two terminal fields in the ipsilateral and three in the contralateral LGN. Presumably, the additional field that we observe contralaterally is layer 6 , which is adjacent to the heavily labeled optic tract and thus not as immediately apparent. In any case, the results of both studies indicate a segregation of the retinogeniculate fibers by eye at birth and, consequently, prior to interlaminar space formation.

Studies in several other species also suggest that retinal afferents begin to segregate well before interlaminar spaces appear. Thus, in the prenatal kitten (Shatz and DiBerardino, 1980), prenatal monkey (P. Rakic, personal communication), postnatal squirrel (C. Cusick and J. H. Kaas, personal communication), and postnatal ierret (Linden et al., 1981), retinal afferents to the LGN begin to segregate prior to the initial appearance of interlaminar spaces. In fact, in both the tree shrew and cat (C. J. Shatz, personal communication), the afferents exhibit a nearly adult laminar pattern prior to the first hints of interlaminar space formation. Results in the ferret (Linden et al., 1981) suggest that final segregation of retinal afferents takes place after interlaminar spaces have reached their mature state. Although more data are required to know when the absolute end point of retinal afferent segregation in other species is reached, the data from all species studied thus far are consistent with the suggestion that retinal afferent segregation begins prior to interlaminar space formation.

A comparison also can be made between the time of development of interlaminar spaces and the time of maturation of cytological features characteristic of individual LGN layers. Although, at birth, the cells within the tree shrew LGN are quite immature in appearance, they frequently do have some cytoplasm as well as Nissl substance. These features represent an intermediate stage of development according to the criteria suggested by La Velle $(1951,1956)$. Nevertheless, it is not until all interlaminar spaces have formed that the cells exhibit the differential staining and size features characteristic of individual LGN layers. Similarly, cells in the human LGN (Hitchcock and Hickey, 1980) acquire morphologies characteristic of the magnocellular and parvocellular layers only after interlaminar space formation is well advanced in those regions of the nucleus. In fact, cells in the human LGN appear to be much less mature when interlaminar spaces begin to form than are those in the tree shrew LGN at a comparable stage of development. Thus, in the human, tree shrew, and possibly the ferret (see Linden et al., 1981) as well, the cytological features characteristic of individual LGN layers develop later than do the interlaminar spaces. These observations suggest that the three events, namely, retinal afferent segregation, interlaminar space formation, and cytological maturation, that characterize LGN layers occur according to different developmental timetables. Thus, the segregation of retinal afferents begins first, followed by the initiation of interlaminar spaces, and finally by the maturation of cytological features characteristic of individual LGN layers.

A comparison of the time of formation of individual interlaminar spaces relative to each other is also of interest since some interlaminar spaces form before others. This variation in time of interlaminar space formation within a single species has not been reported previously. However, apparently, in the human, as in the tree shrew, the formation of the interlaminar spaces adjacent to layers innervated by opposite eyes precedes the formation of those between layers innervated by the same eye, i.e., between the ipsilaterally innervated magno- and parvocellular laminae (T. L. Hickey, personal communication). In both cases, the first spaces to form are most simply related to a functional division between left and right eyes within the nucleus. The formation of the remaining spaces is less easy to define functionally. In the tree shrew, it may be significant, however, that the spaces between layers innervated by the same eye are also the borders of layer 3 , which indirect evidence shows may be functionally unique. For example, the size, connections, histochemical characteristics, and response to deprivation of the cells in the tree shrew layer 3 (Casagrande et al., 1978; Carey et al., 1979; Fitzpatrick et al., 1980; Brauer et al., 1981) are similar to those same features in layers of the cat and galago LGN (LeVay and Gilbert, 1976; Carey et al., 1979; Graybiel and Berson, 1980; Fitzpatrick and Diamond, 1979; Hickey, 1980; Casagrande and Joseph, 1980) which have been shown to contain W- or W-like cells (Wilson and Stone, 1975; Cleland et al., 1976; Wilson et al., 1976; Norton and Casagrande, 1982). Since all interlaminar spaces are dependent for formation upon the continued presence of retinal afferents (Brunso-Bechtold and Casagrande, 1981), temporal differences in the development of interlaminar spaces may relate to differences in the rate of maturation of functionally different pathways (perhaps $\mathrm{W}, \mathrm{X}$, and $\mathrm{Y}$ ) from the retina.

Mechanism of interlaminar space formalion. The results of the present and related studies allow us to address the issue of the causal factors involved in interlaminar space formation. Concerning the role of cellular maturation, we know that removal of both eyes at birth does not prevent LGN cells from developing cytological features characteristic of individual layers, even though 
interlaminar spaces do not form (Brunso-Bechtold and Casagrande, 1981). Furthermore, our observations, as well as those of others which we have just reviewed, indicate that LGN somata are still relatively immature when the interlaminar spaces are in an advanced stage of development. These results suggest that maturation of LGN cells is not the critical event in the initiation of interlaminar space formation.

In contrast to the as yet unclear role of LGN cell maturation, retinal afferents are critical to interlaminar space formation. Nevertheless, the relationship between these events is by no means clear. Retinal afferents in all species start to segregate prior to interlaminar space formation and, in the ferret at least, do not complete segregation until after interlaminar spaces are formed. The question then arises: in what ways are retinal afferents essential to interlaminar space formation? One possibility is that retinal fibers may synapse on immature LGN somata or initial segments of developing dendrites. As a result of the occupation of available synapses by retinal afferents, there would be no synaptic space on more distal portions of developing dendrites for incoming fibers (corticogeniculate or tectogeniculate fibers), some of which travel parallel to the layers and, in adult tree shrews, end predominantly in interlaminar regions (Casagrande, 1974). These fibers then might push between cells in a manner resulting in interlaminar spaces. This hypothesis gains credence from our recent experiments (Brunso-Bechtold and Casagrande, 1982) which demonstrate that, in the tree shrew, as in the monkey (Shatz and Rakic, 1981), corticogeniculate fibers lag behind retinogeniculate afferents in development.

\section{References}

Birecree, E. A., S. Florence, and V. A. Casagrande (1980) Effects of deprivation on cortico-geniculate projections in tree shrew. Invest. Ophthalmol. Vis. Sci. Suppl. 242.

Brauer, K., L. Werner, E. Winkelmann, and H. -J. Luth (1981) The dorsal lateral geniculate nucleus of Tupaia glis: A Golgi, Nissl, and acetylcholinesterase study. J. Hirnforsch. 22: 5974.

Brunso-Bechtold, J. K., and V. A. Casagrande (1980) Early postnatal development of the dorsal lateral geniculate nucleus of the tree shrew. Anat. Rec. 196: 24A.

Brunso-Bechtold, J. K., and V. A. Casagrande (1981) Effect of bilateral enucleation of the development of layers in the dorsal lateral geniculate nucleus. Neuroscience 6: 2579-2586.

Brunso-Bechtold, J. K., and V. A. Casagrande (1982) Laminar development of the lateral geniculate nucleus (LGN) in the tree shrew. Anat. Rec. 202: 215A.

Campbell, C. B. G., J. Jane, and D. Yashon (1967) The retinal projections of the tree shrew and hedgehog. Brain Res. 5: 406-418.

Carey, R. G., D. Fitzpatrick, and I. T. Diamond (1979) Thalamic projections to layer I of the striate cortex shown by retrograde transport of horseradish peroxidase. Science 203: 556-559.

Casagrande, V. A. (1974) The laminar organization and connections of the lateral geniculate nucleus in the tree shrew (Tupaia glis). Anat. Rec. 178: 323 .

Casagrande, V. A., and J. K. Harting (1975) Transneuronal transport of tritiated fucose and proline in the visual pathways of the tree shrew, Tupaia glis. Brain Res. 96: 367-373.

Casagrande, V. A., and R. Joseph (1980) Morphological effects of monocular deprivation and recovery on the dorsal lateral geniculate nucleus in galago. J. Comp. Neurol. 194: 413-426.

Casagrande, V. A., R. Guillery, and J. K. Harting (1978) Differential effects of monocular deprivation seen in different layers of the lateral geniculate nucleus. J. Comp. Neurol. 179: 469486.

Cleland, B. G., W. R. Levick, R. Morstyn, and H. G. Wagner (1976) Lateral geniculate relay of slowly conducting retinal afferents to cat visual cortex. J. Physiol. (Lond.) 255: 299320 .

Cooper, E. R. A. (1945) The development of the human lateral geniculate body. Brain 68: 222-237.

Cowan, W. M., D. I. Gottlieb, A. E. Hendrickson, J. L. Price, and T. A. Woolsey (1972) The autoradiographic demonstration of axonal connections in the central nervous system. Brain Res. 37: 21-51.

Edwards, S. B. (1972) The ascending and descending projections of the red nucleus in the cat: An experimental study using autoradiographic tracing methods. Brain Res. 48: 4563.

Fitzpatrick, D., and I. T. Diamond (1979) The laminar organization of the lateral geniculate body in Galago senegalensis: A pair of layers identified by acetylcholinesterase activity. Brain Res. 170: 538-542.

Fitzpatrick, D., R. G. Carey, and I. T. Diamond (1980) The projection of the superior colliculus upon the lateral geniculate body in Tupaia glis and Galago senegalensis. Brain Res. 194: 494-499.

Gilbert, M. S. (1934) The early development of the human diencephalon. J. Comp. Neurol. 62: 81-116.

Glickstein, M. (1967) Laminar structure of the dorsal lateral geniculate nucleus in the tree shrew (Tupaia glis). J. Comp. Neurol. 131: 93-102.

Graybiel, A. M., and D. M. Berson (1980) Autoradiographic evidence for a projection from the pretectal nucleus of the optic tract to the dorsal lateral geniculate complex in the cat. Brain Res. 195: 1-12.

Harting, J. K., I. T. Diannond, and W. C. Hall (1973) Anterograde degeneration study of the cortical projections of the lateral geniculate and pulvinar nuclei in the tree shrew $(T u$ paia glis). J. Comp. Neurol. 150: 393-440.

Hickey, T. L. (1980) Development of the dorsal lateral geniculate nucleus in normal and visually deprived cats. J. Comp. Neurol. 189: 467-481.

Hitchcock, P. F., and T. L. Hickey (1980) Prenatal development of the human lateral geniculate nucleus. J. Comp. Neurol. 194: 375-411.

Hubel, D. H. (1975) An autoradiographic study of the retinocortical projections in the tree shrew (Tupaia glis). Brain Res. 96: 41-50.

Kalil, R. (1978) Development of the dorsal lateral geniculate nucleus in the cat. J. Comp. Neurol. 182: 265-292.

Laemle, L. K. (1968) Retinal projections of Tupaia glis. Brain Behav. Evol. 1: 473-499.

La Velle, A. (1951) Nucleolar changes and development of Nissl substance in the cerebral cortex of fetal guinea pigs. J. Comp. Neurol. 94: 453-467.

La Velle, A. (1956) Nucleolar and Nissl substance development in nerve cells. J. Comp. Neurol. 104: 175-201.

LeVay, S., and C. D. Gilbert (1976) Laminar patterns of geniculo-cortical projections in the cat. Brain Res. 113: 1-20.

Linden, D. C., R. W. Guillery, and J. Cuechiaro (1981) The dorsal lateral geniculate nucleus of the normal ferret and its postnatal development. J. Comp. Neurol. 203: 189-212.

Moskowitz, N., and C. R. Noback (1962) The human lateral geniculate body in normal development and congenital unilateral anophthalmia. J. Neuropathol. Exp. Neurol. 21: 377382.

Norton, T. T., and V. A. Casagrande (1982) Laminar organiza- 
tion of receptive-field properties in the lateral geniculate nucleus of bushbaby (Galago crassicaudatus). J. Neurophysiol. 47: 715-741.

Norton, T. T., V. A. Casagrande, and S. Sherman (1977) Loss of Y-cells in the lateral geniculate nucleus of monocularly deprived tree shrews. Science 197: 784-786.

Rager, G., R. S. Nowakowski, S. Lausmann, M. Tanaka, and A. Schwaier (1980) The development of the retino-geniculocortical projection in the tree shrew, Tupaia belangeri. Soc. Neurosci. Abstr. 6: 662 .

Rakic, P. (1977a) Genesis of the dorsal lateral geniculate nucleus in the rhesus monkey: Site and time of origin, kinetics of proliferation, routes of migration and pattern of distribution of neurons. J. Comp. Neurol. 176: 23-52.

Rakic, P. (1977b) Prenatal development of the visual system in rhesus monkey. Philos. Trans, R. Soc. Lond. (Biol.) 278: 245260.

Shatz, C. J., and A. C. DiBerardino (1980) Prenatal development of the retino-geniculate pathway in the cat. Soc. Neurosci. Abstr. 6: 485.

Shatz, C. J., and P. Rakic (1981) The genesis of efferent con- nections from the visual cortex of the fetal rhesus monkey. J. Comp. Neurol. 196: 287-308.

Sherman, S. M., R. W. Guillery, J. H. Kaas, and J. K. Sanderson (1974) Behavioral electrophysiological and morphological studies of binocular competition in the development of the geniculo-cortical pathways of cats. J. Comp. Neurol. 158: 118.

Snyder, M., and I. T. Diamond (1968) The organization and function of the visual cortex in the tree shrew. Brain Behav. Evol. 1: 244-288.

Tigges, J. (1966) Ein experimenteller beitrag zum subkortikalen optischen system von Tupaia glis. Folia Primatol. (Basel) 4: 103-123.

Wilson, P. D., and I. Stone (1975) Evidence of W-cell input to the cat's visual cortex via the C-laminae of the lateral geniculate nucleus. Brain Res. 92: 472-478.

Wilson, P. D., M. H. Rowe, and J. Stone (1976) Properties of relay cells in cat's lateral geniculate nucleus: A comparison of W-cells with X-and Y-cells. J. Neurophysiol. 39: 1193-1209.

Zilles, K. J. (1978) Ontogenesis of the visual system. Adv. Anat. Embryol. Cell Biol. 54: 5-138. 